\title{
Assessing Readiness of Community Pharmacists to Perform and Document Medically Billed Clinical Services
}

\author{
Matthew Elamparo; PharmD ${ }^{1}$; Amy Jay; PharmD2; Tara Pfund; PharmD²; Julie Akers; PharmD, BCACP ${ }^{3}$
}

${ }^{1}$ Fred Meyer and Washington State University; ${ }^{2}$ Fred Meyer; ${ }^{3}$ Washington State University

\begin{abstract}
Objectives: To assess the readiness of community pharmacists in documenting and performing medically billed clinical services. Additionally, the preferred training method of community pharmacists was evaluated.

Methods: This study surveyed practicing pharmacists in a chain community pharmacy in Washington State via a voluntary, anonymous online survey through Qualtrics. Data collected in the survey measured each pharmacist's self-perceived ability to perform examinations and to gather and document patient subjective and objective information compliant with medical billing requirements. In addition, questions evaluated the training methods pharmacists preferred in the community setting. The data was aggregated and analyzed utilizing descriptive statistics to assess pharmacists' self-perceived baseline understanding regarding documenting and performing medically billed clinical services as well training preferences of the pharmacists surveyed.

Results: Pharmacists in the study had a generally higher perceived ability in performing past medical history, medical decision-making and clinical documentation of a medical visit. In contrast, pharmacists in the community setting had a lower perceived ability performing physical assessment criteria in a medically billed clinical visit. This study indicated that pharmacists in the community setting preferred live small-group training on future medical billing.

Conclusion: Pharmacists have some of the skills necessary to perform and bill clinical visits; however, there is room for improvement in particular areas where pharmacists have a lack of training and experience.
\end{abstract}

Keywords: Medical billing, Pharmacist providers

\section{Introduction}

It is evident there is a current shortage of primary care providers nationwide. ${ }^{1}$ Patients may have difficulty scheduling timely appointments with providers in the outpatient setting due to this shortage. Many patients look to pharmacists in the outpatient and community setting to fill this void by seeking advice on self-care options as well as through the provision of clinical services, as legislation allows. However, there is often little to no reimbursement for the cost of the clinical service. The lack of reimbursement by insurance for medical services provided by pharmacists has been a major barrier to the sustainability and expansion of these services. ${ }^{2}$

In Washington State, legislation was established requiring that pharmacists be included in commercial medical insurance plans as providers. This provides pharmacists in Washington state an avenue to bill for the clinical services they provide. However, there is limited research available evaluating pharmacists' abilities to perform and document medically billed clinical services. Ultimately, this study provides insight on pharmacists' perceived capabilities providing clinical services according to medical billing standards - with the potential of billing for services provided.

Corresponding author: Julie Akers; PharmD, BCACP Clinical Assistant Professor

WSU College of Pharmacy, 205 E Spokane Falls Blvd Spokane, WA 99202; Email: Julie.akers@wsu.edu

\section{Objective}

The primary objective of this study was to assess the readiness of community pharmacists in performing and documenting medically billed clinical patient care visits according to Center of Medicare and Medicaid (CMS) standards. Additionally, the study evaluated the preferential methods in which the community pharmacists surveyed would prefer to be trained to enhance understanding and compliance with CMS standards.

\section{Method \\ Design \\ The primary study aim was to evaluate the perceived ability of community pharmacists in performing and documenting medically billed clinical services according to CMS standards. With legislation allowing pharmacists to bill clinical services through private medical insurance in Washington State, the goal was to assess the readiness of pharmacists to meet the standards. The study evaluated community pharmacists from a major community pharmacy chain in Washington State. Data was collected related to the pharmacists type of work experience, degree and qualifications, and range of graduation year. The evaluation was conducted via a voluntary anonymous survey distributed electronically to pharmacists in the study.}

The secondary study aim was to evaluate the preferred training preferences of the community pharmacists surveyed and if additional education on performing and documentation of clinical services would be necessary. Additionally, the survey evaluated preferences gathered for pharmacists that met the inclusion criteria of the study. 
The study was conducted by a PGY1 Community Pharmacy Residency Program resident, in collaboration with the residency research project team and was deemed exempt by the Washington State University IRB.

\section{Intervention}

The study utilized an anonymous online survey to evaluate the perceived ability of pharmacists to document and perform medically billed clinical services according to CMS standards. The survey was proctored via Qualtrics online survey platform and distributed via an email listserv to target participants. Within the email, participants were introduced to the study and provided with the link to the Qualtrics survey. Additionally, supplementary material was provided for study participants that defined specific terminology that survey participants may not have been familiar with. Participants were given three weeks to complete the survey.

Survey question formatting included multiple choice, Likert scale (1-5 scale) and free response format. Primary study aim questions included assessing pharmacists' perceived ability to perform and document past medical history $(\mathrm{PMH})$, physical assessment, and medical decision-making (MDM) criteria. Secondary aim questions included preference of written, online, and/or live training sessions.

The survey queried each pharmacist's level of educational attainment as well as previous pharmacy experience. This data was utilized in subgroup analysis to descriptively depict differences between pharmacists' group scores based on education and/or prior experience.

\section{Outcome measures and analysis}

To assess the participants' perceived ability, survey results were exported electronically from Qualtrics to Microsoft Excel to further evaluate the data based on pharmacist subgroup analysis.

The method of analysis of this study utilized descriptive statistics to evaluate average scores and differences from average between subgroups versus the entire study population. The data was group into subcategories as follows: agree to strongly agree (4-5 on Likert scale), neutral (3 on Likert scale) and disagree to strongly disagree (1-2 on Likert scale). Training preference data was gathered and evaluated descriptively based on percentage of participants that preferred a particular training method.

\section{Results}

Survey results from participating community pharmacists ( $\mathrm{n}=$ 86) showed varying perceived ability to perform tasks relevant to medical billing of clinical services. When asked to evaluate their level of confidence in ability to evaluate past medical history, $82 \%$ of subjects $(n=70)$ agreed or strongly agreed regarding gathering patient medical history, while $12 \%(n=10)$ and $6 \%(n=5)$ of participants were neutral and disagreed or strongly disagreed, respectively. Furthermore, $43 \%(n=37)$ of participants agreed or strongly agreed in being familiar performing physical assessments, while $40 \%(n=34)$ and $17 \%$ $(n=15)$ of participants were neutral and disagreed or strongly disagreed, respectively. In regards to medical decision-making criteria, $80 \%$ of pharmacists agreed or strongly agreed with having familiarity with performing this component, while $12 \%$ $(n=10)$ and $8 \%(n=7)$ felt neutral and disagreed or strongly disagreed, respectively. (Figure 1)

When evaluating the perceived ability to document clinical services according to CMS medical billing standards, $62 \%(n=53)$ of participants agreed or strongly agreed with having familiarity performing this component, while $24 \%(n=24)$ and $14 \%(n=12)$ were neutral and disagreed or strongly disagreed, respectively.

When evaluating different subgroups, pharmacists with doctorate degrees had higher than average Likert scale scores in $\mathrm{PMH}$ and $\mathrm{MDM}$ categories versus pharmacists with a Bachelor of Science in pharmacy degrees. Additionally, pharmacists with inpatient experience, those with additional clinical certifications (such as BCPS, CDE, BCACP, etc.), those that graduated before 2007, and those with a Bachelor of Science in pharmacy degree had higher than average Likert scale scores for perceived ability in performing physical assessment tasks relevant to medically billing clinical services. In regard to medical decision-making, those with ambulatory care experience and inpatient experience had higher than average Likert scale scores in comparison to the rest of the participants. When participants perceived ability to document clinical services was assessed, those with residency training and pharmacists with clinical certifications had higher than average Likert scale scores. Additionally, those graduating prior to 2007, those with staffing experience in the community setting, and those with a Bachelor of Science in pharmacy degree had lower than average scores in perceived ability to document clinical visits. (Figure 2)

The study also evaluated participants' training preferences for future medical billing training sessions, with the ability to select more than one training option. Survey results revealed $68 \%$ $(n=58)$ of participants preferred small group training, 37\% $(n=32)$ preferred online web-based learning, 20\% ( $n=17)$ preferred training via policies and procedures, 20\% ( $n=17)$ preferred large group training, and $18.82 \%(n=16)$ preferred online live training. (Figure 3 )

\section{Discussion}

There are many factors affecting a pharmacist's perceived ability to perform and document medically billed clinical services. Ones perceived ability to perform these services is critical in the future of pharmacy medical billing, as it provides baseline knowledge of what pharmacists in the community setting are generally comfortable performing. With this baseline assessment in perceived ability, a training plan can then be developed to provide pharmacist's skills in areas they 
have a lack of confidence performing. Furthermore, it provides information to generate a learning and training plan that allows pharmacists to gain knowledge and skills on the components they are less comfortable performing.

In regards to the level of education attained, those who graduated more recently through doctorate of pharmacy programs were shown to have an increased perceived ability to perform tasks relevant to medically billing clinical visits. This could be due to the increased emphasis on performing clinical assessments in doctor of pharmacy program curriculum. It could be inferred that academic curriculum in these programs provides adequate preparation to give pharmacists the tools to perform as providers in the community setting. Additionally, those with higher education attained have a lower perceived ability when it comes to physical assessment. This could potentially be attributed to those graduating more recently and those with Doctor of pharmacy degrees having a greater understanding about the complexity of physical assessment in the medical billing process, thus altering their perceived ability toward being less comfortable.

In comparison to other major areas of the medical billing process, Pharmacists were least comfortable performing the physical assessment aspect of a clinical visit. This suggests that physical assessment competency should be emphasized when training pharmacists in the future. However, an alternative for pharmacists less comfortable performing the physical assessment is to perform and bill for two out of three major components (PMH and MDM), which is acceptable for followup patients under CMS standards. Only in new patients is it required under $\mathrm{CMS}$ standards to perform $\mathrm{PMH}, \mathrm{MDM}$, and physical assessment criteria.

As previously stated, perceived ability data and training preferences gathered from this study can be utilized to form specific training based on the topics reported with belowaverage perceived ability. This provides an opportunity to fill the knowledge gaps pharmacists may potentially have, thus allowing them to be competent in performing and documenting medically billed clinical services.

An ideal follow up to this study would be to assess pharmacists' actual ability to perform and document medically billed clinical services. This could be accomplished by providing participants with mock patient cases to assess the pharmacists' ability to perform and document a clinical visit to CMS standards. Data gathered could be compared to results from this study to evaluate perceived ability in comparison to their actual ability to perform these services.

\section{Limitations}

A major limitation of this study was that results were based solely on the perceived ability of pharmacists to perform and document clinical services. This study did not assess their actual ability to perform and document services, which is eventually the quality measure in which pharmacists will be assessed in the practical setting as providers. Additionally, the study surveyed pharmacists from a single community pharmacy chain within a limited geographical region.

\section{Conclusion}

Ultimately, pharmacists have the potential to provide billable clinical services in the community pharmacy setting depending on current legislation in the state of practice. This study demonstrated that pharmacists have a perceived ability in performing some skills required to perform and document medically billable clinical visits. The pharmacists surveyed had a perceived ability in performing past medical history, documentation, and the medical decision making process. However, pharmacists had a lack of perceived ability performing physical assessment in the medical billing process. In addition, pharmacists indicated that they preferred small group live sessions for future medical billing training. These findings provide insight to the current status of pharmacists with regards to the performing and documentation of medically billed clinical services. Ultimately, pharmacists have some skills necessary to perform and bill clinical visits; however, there is room for improvement in some areas where pharmacists may lack training or experience.

Disclosure: Tara Pfund, PharmD and Amy Jay, PharmD are employees of Fred Meyer, which is the chain pharmacy in which this study was conducted. Matthew Elamparo, PharmD was a PGY-1 Community Pharmacy Resident and employee of Fred Meyer at the time this research was conducted. Any potential conflicts of interest were resolved during the Institutional Review Board (IRB) process.

This research did not receive any specific grant from funding agencies in the public, commercial, or not-for-profit sectors.

Previous Presentations: Summary findings of this research were presented as a poster presentation at the annual American Pharmacists Association (APhA) conference in Nashville, TN March 2018 and the Northwest Convention in Coeur D'Alene, ID June 2018. It was presented as a podium presentation at the Northwestern States Regional Conference in Portland, OR May 2018.

\section{References}

1. Mann, S. (2016). New AAMC research confirms looming physician shortage. AAMC Report. 2016. Accessed 14 Feb 2018.

2. Traynor, K. (2015). Pharmacists praise new Medicare billing opportunities: provider status still critical they say. American journal of health-system pharmacy: AJHP: official journal of the American Society of Health-System Pharmacists, 72(2), 91. Accessed 14 Feb 2018. 
Figure 1: Comparison of pharmacists' perceived ability performing tasks relevant to medically billed clinical services - including gathering past medical history data, physical assessment, generating medical decision-making (MDM) criteria, and the proper documentation of clinical services provided.

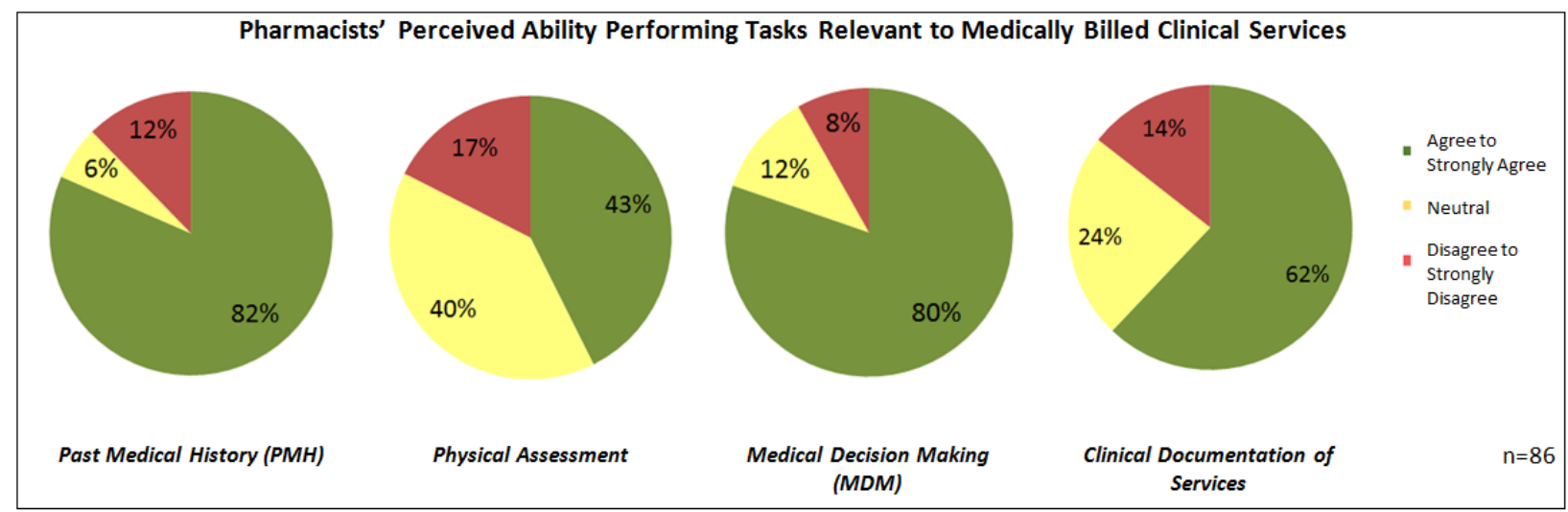

Figure 2: Comparison of pharmacists' perceived ability performing tasks relevant to medically billing clinical services by training and experience.

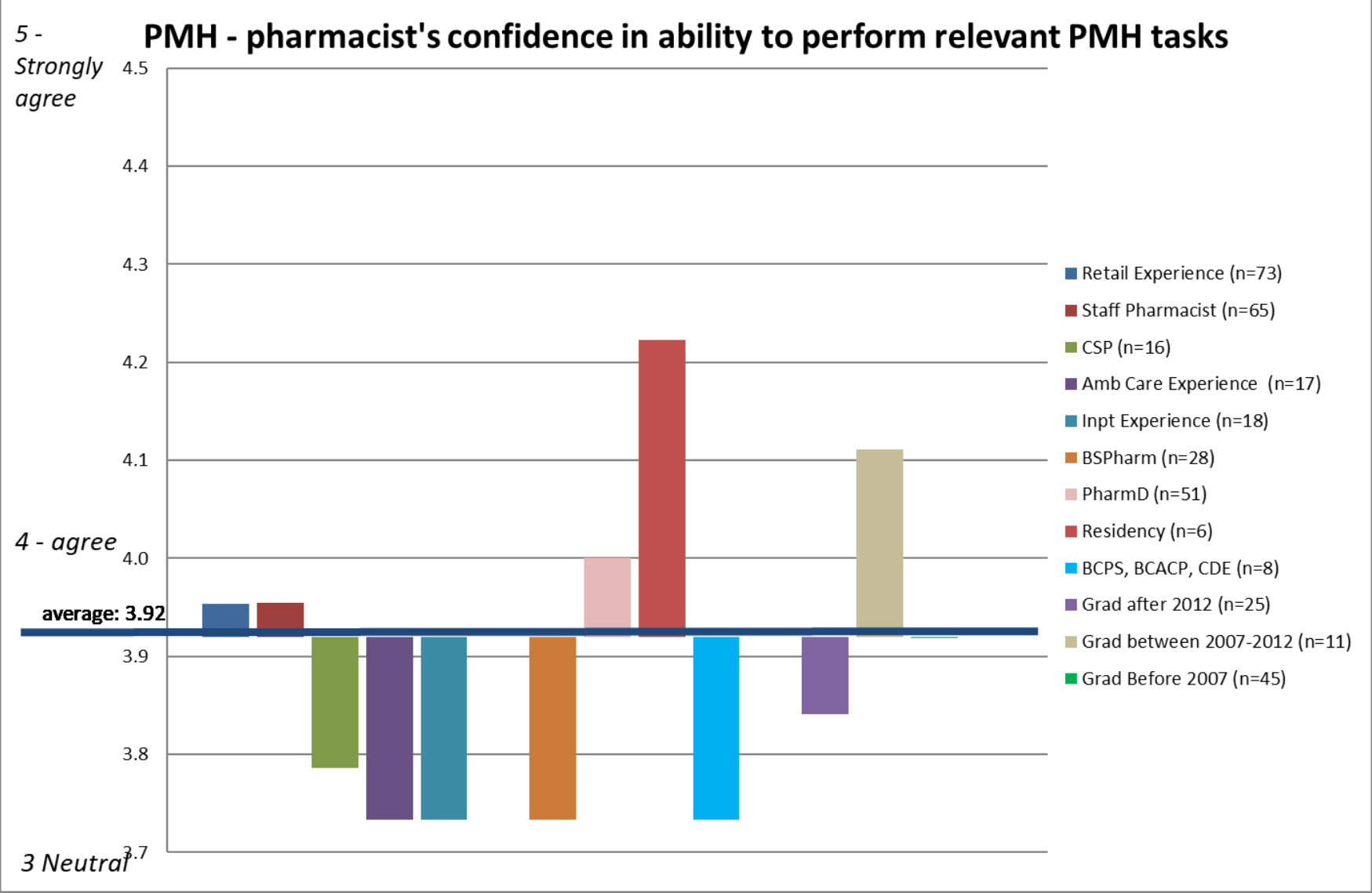



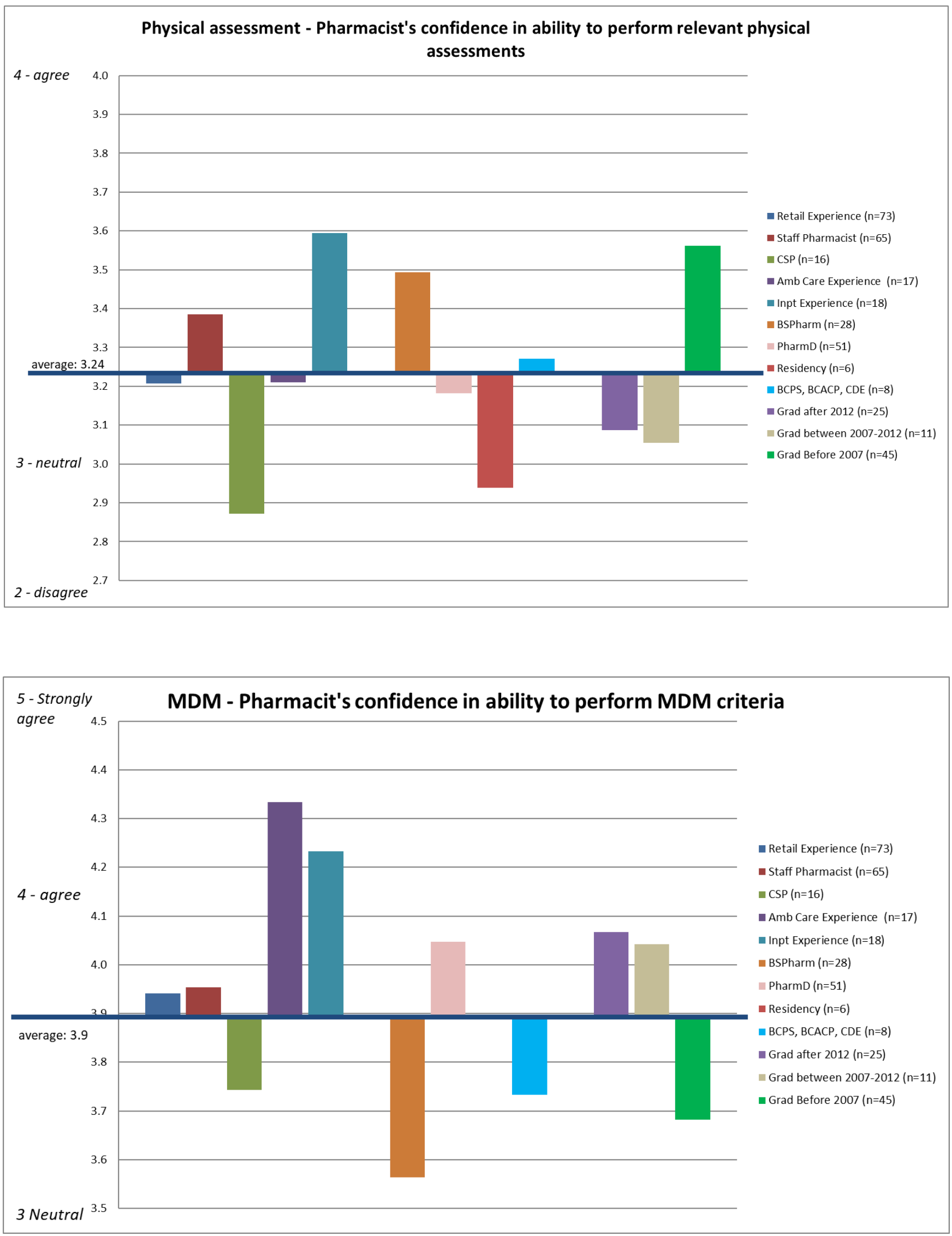
Figure 3: Preferences of study participants' preferred method of training relevant to performing and documenting medically billed clinical services (more than one option could be selected).

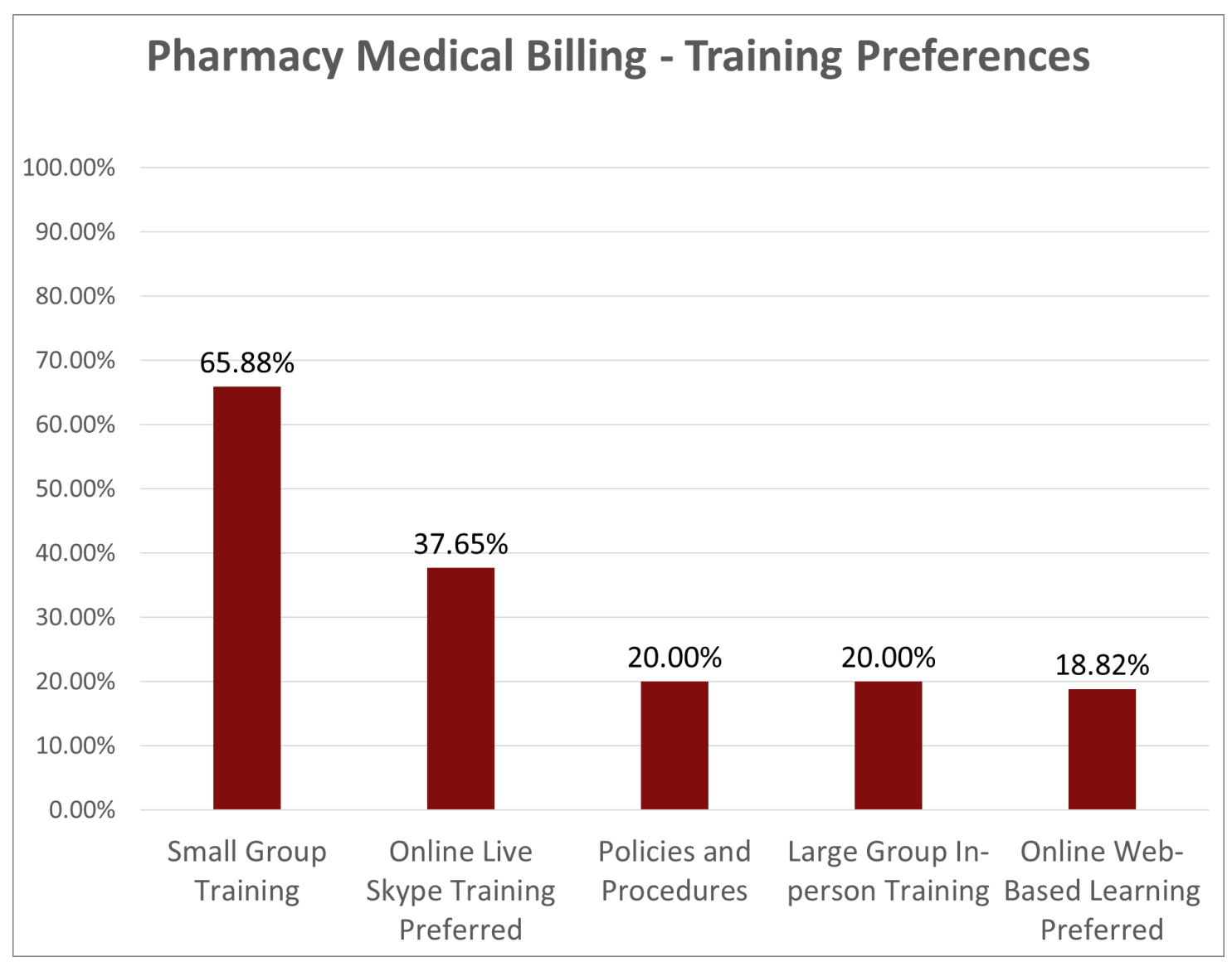

